\section{Mechanical properties of the human stomach under uniaxial stress action}

\author{
Grzegorz Rotta ${ }^{1, *}$, Jarosław Kobiela ${ }^{2}$, \\ Szymon Grymek ${ }^{1}$ and Magdalena Karczewska ${ }^{1}$ \\ ${ }^{1}$ Institute of Medical Engineering, Department of Machines and \\ Vehicles Design, Faculty of Mechanical Engineering, \\ Gdansk University of Technology, G. Narutowicza Str, 11/12, \\ 80-233 Gdansk, Poland \\ ${ }^{2}$ Department of General, Endocrine and Transplant Surgery, \\ Medical University of Gdansk, Smoluchowskiego Str, \\ 17, 80-214 Gdansk, Poland
}

The aim of this study was to estimate the range of mechanical properties of the human stomach in order to use the collected data in numerical modelling of surgical stapling during resections of the stomach. The biomedical tests were conducted in a self-developed tensile test machine. Twenty-two fresh human stomach specimens were used for the experimental study of its general strength. The specimens were obtained from morbidly obese patients on whom sleeve gastrectomy was performed. Finally, data on mechanical properties of the stomach wall with detailed analysis of anatomical regions of the stomach have been presented.

Keywords: Human stomach, sleeve gastrectomy, uniaxial stretch, mechanical properties.

MECHANICAL properties of human tissues are important variables used in computer modelling of surgical procedures, optimization of operative techniques and the designing of surgical equipment. They are also used in virtual reality surgical simulations ${ }^{1-3}$. Obtaining various types of input data, depending on the mechanical model used is essential for these applications.

Currently, one of the most dynamically developing areas of medicine is stomach surgery, which is mostly due to the obesity epidemic and the growing popularity of bariatric surgeries. These surgical procedures, largely based on resection of the stomach, are meant to decrease energy supply, reduce body mass and improve the quality of life and survival rate of patients. The procedures are usually carried out using laproscopic stapling devices surgical staples. Mechanical properties of stapler suturing are of significant importance due to the potentially fatal complications caused by leaks from the staple line ${ }^{4-7}$. In this case, determining material parameters of stomach tissue may be useful in efforts to optimize the quality of mechanical suturing by taking into account different properties of tissues located along the staple line.

The aim of this study was to estimate the range of mechanical properties of the human stomach. Preparation of test samples, experimental procedure and results

*For correspondence. (e-mail: grotta@pg.edu.pl) presented as a stress-strain characteristic are discussed in this communication. The collected data can be employed in numerical modelling of surgical procedures, e.g. stapling during resections of the stomach. Additionally, the obtained results were compared to those collected from rat and rabbit stomachs tested by Zhao et al. ${ }^{8}$ and presented as an exponential stress-strain Fung relation ${ }^{9}$.

Fresh human stomach specimens were used for an experimental study of the general strength of these organs. The specimens were obtained from morbidly obese patients on whom sleeve gastrectomy was performed. This is one of the most common bariatric procedures based on a resection of majority of the stomach, which leaves only a sleeve tube along the lesser curvature. Twenty-two fresh human stomach specimens, both male and female, were used in this study. The weight of the patients ranged between 120 and $200 \mathrm{~kg}$ (BMI $>35 \mathrm{~kg} / \mathrm{sq} . \mathrm{m}$ ), and their age ranged between 18 and 56 years. All the stomach specimens were transported from the operating room to the laboratory within $5 \mathrm{~min}$ after resection and tested within an hour of the process. After resection and during testing the specimen was stored in moisten gauze. Therefore, it was hypothesized that the studied human stomach specimens displayed qualities approximating those of a living organ. Each of the stomach specimens was cut into strips (about 10-30 mm wide, to obtain five or six test samples from each organ) that represent all anatomical regions of the stomach, i.e. fundus, body and antrum.

The biomedical tests were conducted in a self-developed tensile test machine consisting of a ball screw unit with a roller guide cart driven by a stepper motor, two vice holders, a force transducer and a video extensometer system (Figure 1). Calibration of the transducer showed that, for the load range used during the studies $(0-50 \mathrm{~N})$, the force error was less than $2 \%$.

The measurement of deformations was carried out by the non-contacting video extensometer method. The stretching process was recorded by two cameras positioned perpendicular to the sample (Figure 2). Camera no. 1 recorded the extension and change in width, i.e. narrowing of the sample and the current reading of force, while camera no. 2 recorded changes in thickness of the sample.

The stomach specimens were divided into individual test samples (Figure 3). The dimensions of these samples differed within a wide range: length $L=100-120 \mathrm{~mm}$ and width $B=10-30 \mathrm{~mm}$; their thickness depended on the anatomy of each particular organ and varied between $G=2$ and $6 \mathrm{~mm}$. The large variation in length and width of the test samples was proportional to the size of stomach specimen. Therefore, the dimensions were proportional to the size of the stomach. It was assumed that each organ would provide five test samples when dividing the stomach transversely (i.e. perpendicular to the greater curvature). Dividing along the longitudinal axis of the stomach (longitudinal excision) provided six test samples. 
The test samples obtained from the greater curvature were relatively long and, for this reason, divided into two parts. The width of each test sample was also influenced by the size and number of stomach mucosal folds. When the mucosal folds were relatively large and deep, wider test samples were excised, even up to $30 \mathrm{~mm}$, in order to balance the cross-sectional stress distribution.

The test samples were mounted with two clamping devices (Figure $3 e$ ). Two markers at $30 \mathrm{~mm}$ distance were glued to the test sample in order to estimate elongation. The tests were carried out in an air-conditioned room, at the temperature of $20^{\circ} \mathrm{C}$ and in an environment with $60 \%$ humidity.

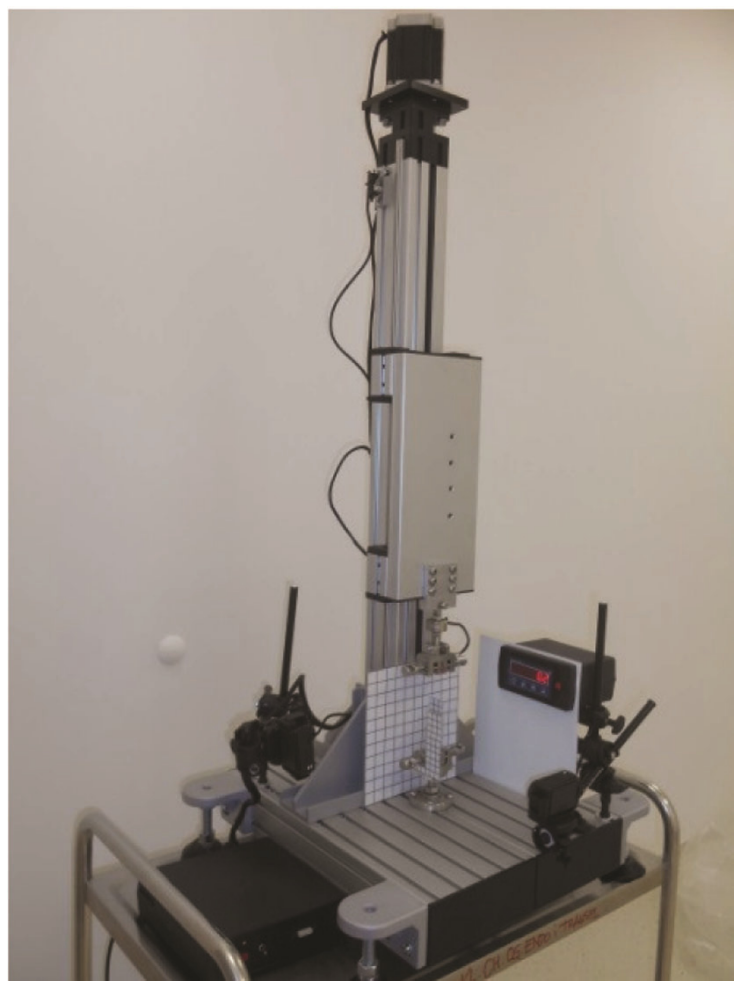

Figure 1. General view of the tensile test machine.

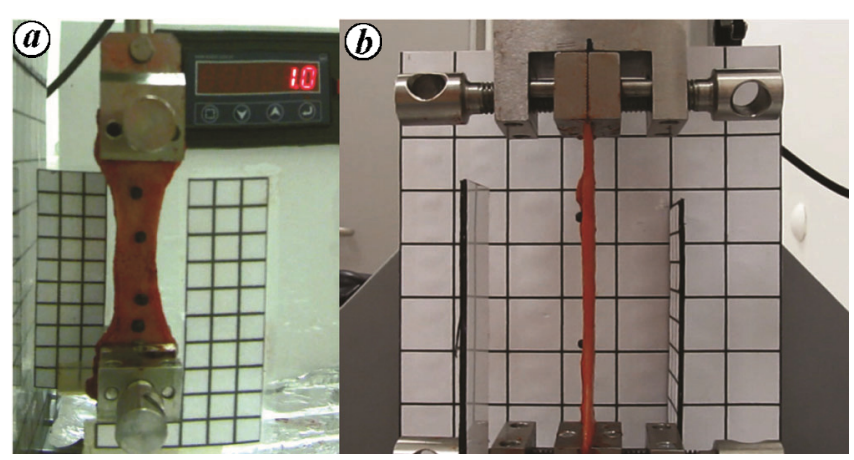

Figure 2. Images used in the video extensometer measurements: $\boldsymbol{a}$, View from camera no. 1 - logs: extension and width of the sample, value of the force (grid control $30 \times 100 \mathrm{~mm}$ ). $\boldsymbol{b}$, View from camera no. 2 - recorded: thickness of the sample; rear of the auxiliary grid control with a side of $20 \mathrm{~mm}$.
Following their mounting via the clamps, test samples were stretched at a constant speed of $0.4 \mathrm{~mm} / \mathrm{s}$. The selected speed was similar to the literature data ${ }^{8,10}$, and has been considered to not influence the results in tension processes $^{11-13}$. On the other hand, one should take note of the fact that these values are not entirely consistent with reality. Propagation velocity of contraction waves in the stomach can achieve a value between 4 and $11 \mathrm{~mm} / \mathrm{s}$, depending on the organism ${ }^{14}$. Other authors claim that propagation velocity can reach much lower values ${ }^{15,16}$, i.e. $2-3 \mathrm{~mm} / \mathrm{s}$. Thus, the expansion rates used by all the authors seem to be much lower than in reality. The nature of deformations during contraction waves in the stomach is different from those during tensile testing. Thus, they cannot be directly compared with regard to deformation velocity.

The test samples did not go through the preconditioning process because they were fresh; instead, the samples were pre-stressed to a force of $0.5 \mathrm{~N}$. During the tests, the stretching process was recorded by two cameras located perpendicular to the sample and to each other. The first camera recorded elongation of the measured segment, sample breadth and reading of the applied force visible on a display, while the second camera recorded thickness of the test sample. The stretching was continued until the test sample was torn, which allowed location of the tear initiation and tearing mechanism to be observed. However, the force required for setting maximum stress levels was accepted as the maximum value which, once exceeded, initiated decrease in the tensile force. This usually took place at the moment of a clear loss of material cohesion at the location of tear.

All the results were presented in the stress-stretch ratio relationship. Stress in test samples was computed as engineering stress $\sigma(\mathrm{MPa})$ using the formula

$$
\sigma=F / A_{0},
$$

where $F$ is the recorded tension force $(N)$ and $A_{0}$ is the initial cross-sectional area $\left(\mathrm{mm}^{2}\right)$.

Cross-sectional area of test samples was calculated as

$$
A_{0}=W_{0} \cdot h_{0},
$$

where $W_{0}$ is the initial width $(\mathrm{mm})$ and $h_{0}$ is the initial thickness (mm).

The width and wall thickness of the samples were averaged from five measurements along the length of the samples between the markers. In order to unify all imagebased measurements, dimensions were taken from outer borders of the sample's view. However, it should be noted that taking the outer dimensions from the analysed images could result in slightly overestimating the total cross-sectional area, mainly due to irregular nature of mucosal folds. Stretch ratio $\lambda$ was calculated as

$$
\lambda=L_{\mathrm{F}} / L_{0},
$$


(a)

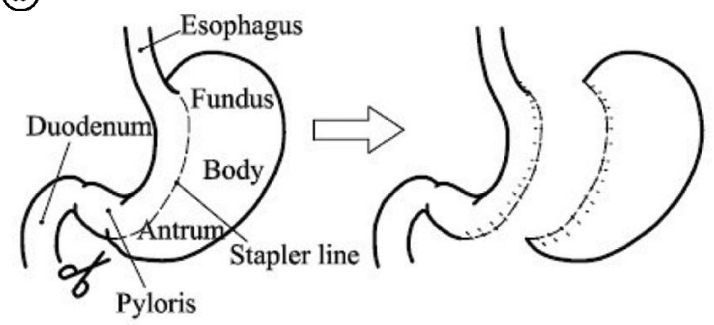

(b)

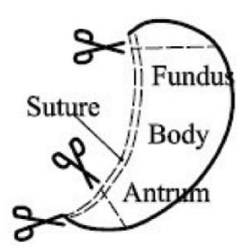

(c)

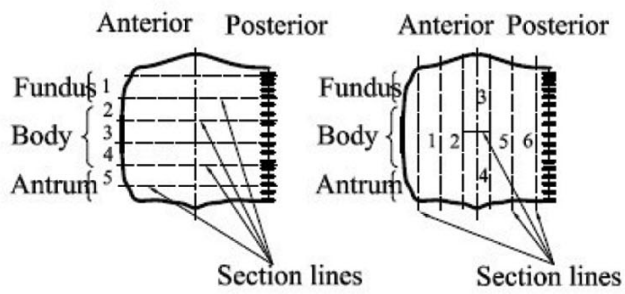

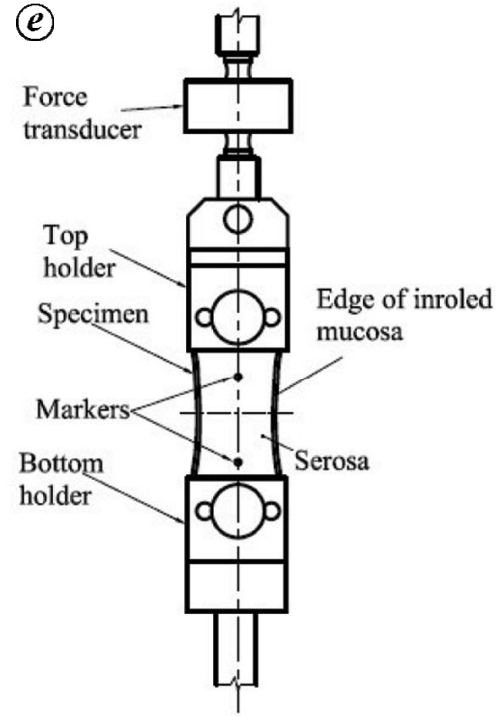

Figure 3. Excision of the test samples. a, Extent of resection during sleeve gastrectomy. $\boldsymbol{b}$, Initial cuts - removal of unnecessary parts of resected stomach. $\boldsymbol{c}$, Preparation of circumferential test samples. $\boldsymbol{d}$, Preparation of longitudinal test samples. $\boldsymbol{e}$, Test sample fixed in holders.
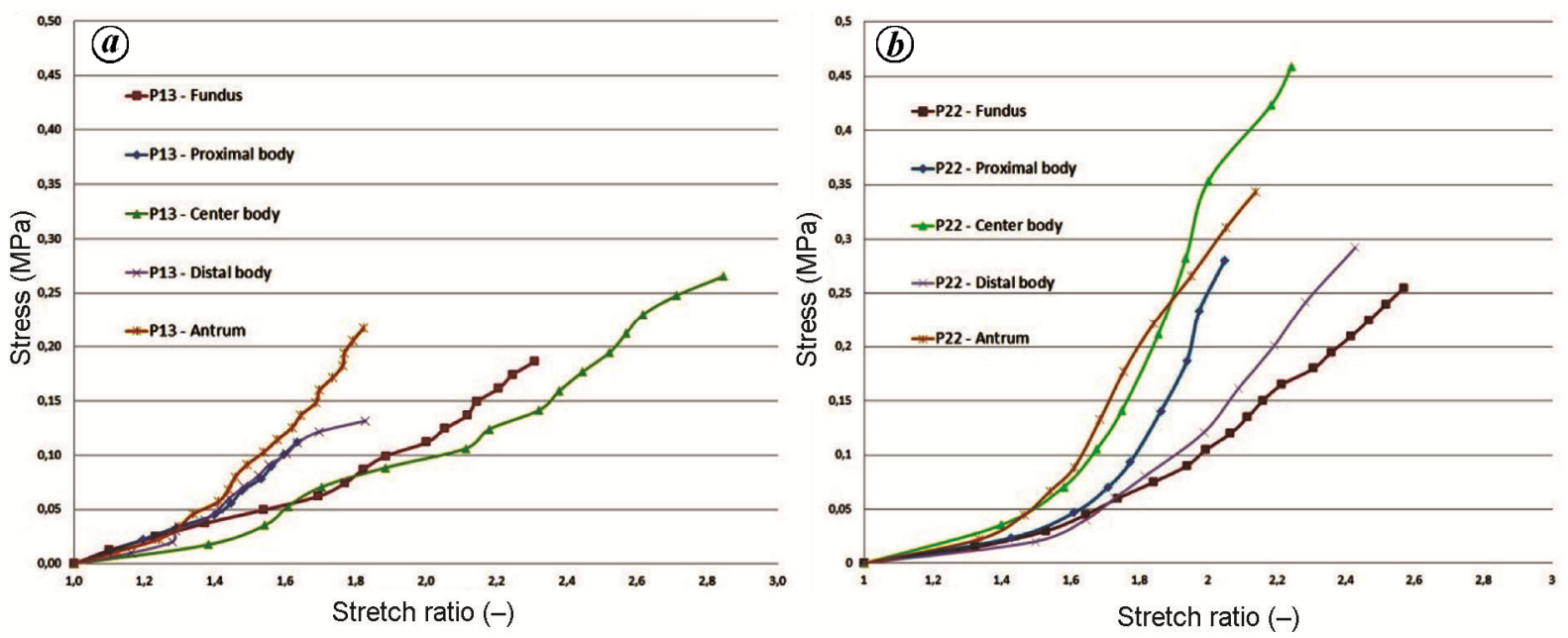

Figure 4. Stress-stretch ratio curves of test samples taken from different locations in two selected stomach specimens in the case of transversely cut test samples: $\boldsymbol{a}$, from stomach no. $13 ; \boldsymbol{b}$, from stomach no. 22.

and it was equivalent to engineering strain $\varepsilon$, because

$$
\varepsilon=\left(L_{\mathrm{F}}-L_{0}\right) / L_{0}=\lambda-1
$$

where $L_{0}$ is the initial length of test samples (distance between the markers) and $L_{\mathrm{F}}$ is the recorded elongation length.

Thus, in order to minimize the amount of data, mechanical characteristics have been mainly presented here as a stress-stretch ratio relationship.

The resultant stress-stretch characteristics can be estimated with an exponential function proposed by Fung $^{9}$

$$
\sigma=b \cdot\left(\mathrm{e}^{(a \cdot(\lambda-1))}-1\right)=b \cdot\left(\mathrm{e}^{(a \cdot \varepsilon)}-1\right)
$$

and consequently Young's modulus is defined as

$$
E(\sigma)=\frac{\mathrm{d} \sigma}{\mathrm{d} \lambda}=\frac{\mathrm{d} \sigma}{\mathrm{d} \varepsilon}=a \cdot(\sigma+b)
$$

Constants $a$ and $b$ were estimated by a curve-fitting procedure solved in a free programing language software, Octave 4.2.0 (https://www.gnu.org/software/octave/). Statistica v. 11 (StatSoft Inc.) was used for statistical analysis. One-way analysis of variance (ANOVA) and $t$ tests were used for comparison of mechanical parameters 


\section{RESEARCH COMMUNICATIONS}

of the tested samples. The results were considered significant for $P<0.05$, and were expressed as mean $\pm \mathrm{SD}$.

The tests consisted of exposing the obtained samples to axial stretching. In total, 22 stomach specimens obtained from sleeve gastrectomy resection performed in morbidly obese patients were examined. Five to six specimens were excised from each stomach, which provided in total over 100 strip-shaped test samples.

Due to the previously mentioned dependence of mechanical properties on the direction of test sample excision $^{8,9,13}$, the study included tests on samples which were excised in two different directions: longitudinally along the stomach, i.e. parallel to the greater curvature and transversely across the stomach, i.e. perpendicular to the greater curvature. The thickness of a specimen depends on its location within the stomach $\left(h_{\text {mean }} \pm \mathrm{SD}\right)$ : fundus $-3.57 \pm 0.80 \mathrm{~mm}$, proximal body $-3.73 \pm$ $0.90 \mathrm{~mm}$, centre body $-3.70 \pm 1.10 \mathrm{~mm}$, distal body $4.25 \pm 1.18 \mathrm{~mm}$ and antrum $-4.24 \pm 1.39 \mathrm{~mm}$. The obtained mechanical results were presented in $\sigma-\lambda$ arrangement. On all the diagrams in this communication, the series that are described as Px signify the respective number of the tested specimen. Figure 4 demonstrates the actual stress-stretch characteristics for two selected stomach specimens, in which the test samples were excised transversely, i.e. across the stomach. One may notice a large spread of characteristics, even in the case of test samples obtained from the same stomach specimen.

Figure 5 shows an example of large spread of the characteristics of test samples cut from the same region in each tested specimens and Figure 6 shows spread of the results for each specific location of the stomach samples. It may be noted that the maximum engineering stress levels usually do not exceed $0.4 \mathrm{MPa}$, while stretch ratio values oscillate between 1.2 and 4.3. Table 1 shows the average values of stress and stretch.

Figure 7 shows typical characteristics of the test samples which were excised along the greater curvature of the stomach. In this case, in comparison with the test samples which were cut out across the stomach, greater tensile strength and stiffness were observed in the test samples. The maximum stress values reached $\sigma=0.62 \mathrm{MPa}$, while the levels of stretch were significantly lower in comparison to test samples cut transversally and were in the range $\lambda=1.2-1.8$. Table 1 shows the mean values of stress and strain for all of the test samples.

Analysis of variance showed that there were no statistically significant differences among the samples cut transversely for both strength $P>0.9$ and strain $P>0.9$. In the case of longitudinally cut samples, similar results were noticed: for strength $P>0.75$ and for strains $P>0.9$. However, $t$-tests showed significant differences of mechanical properties between samples cut transversely and longitudinally $(P<0.05)$, which confirmed anisotropy of the stomach tissue. Figure 8 shows the difference between the coefficients $a$ and $b$ that are calculated during curve fitting eq. (5). Kruskall-Wallis tests showed statistically significant differences $(P<0.05)$ between transverse and longitudinal $a$ coefficients and no differences $(P>0.08)$ between $b$ coefficients.

In the available literature, studies made to determine mechanical characteristics of stomach walls are based on experimental measurements carried out on animal or human cadaver tissues. Experiments carried out on rat and rabbit stomachs demonstrated variable characteristics of mechanical properties depending on the location and direction of the tensile load applied to the test samples. It constituted the basis for anisotropy analysis of swine stomach walls, which are similar in structure to the human organs ${ }^{9}$. Biomechanical tests of the swine stomach

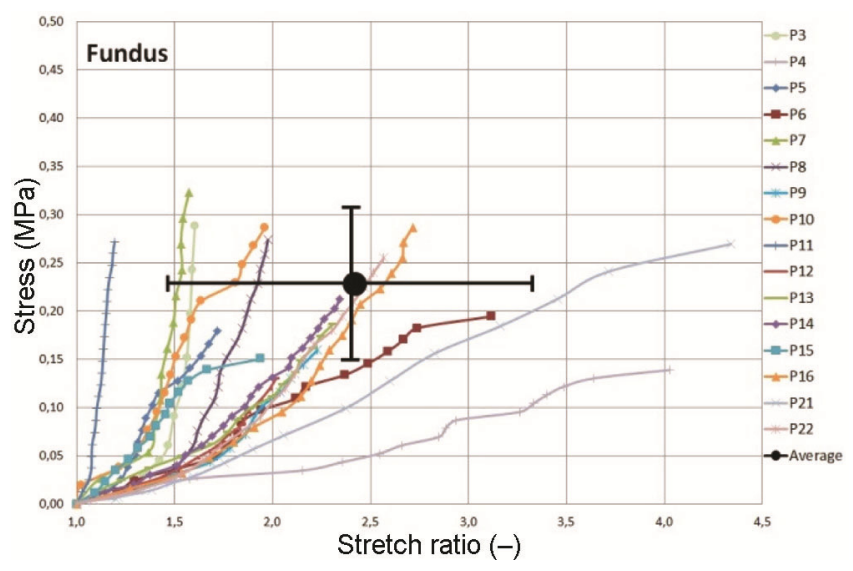

Figure 5. Scatter of the engineering stress-stretch ratio curves of transversally cut test samples from the same location (in this example fundus) on the stomach, but taken from different specimens $(0.23 \pm 0.06 \mathrm{MPa}$ and $2.41 \pm 0.83)$.

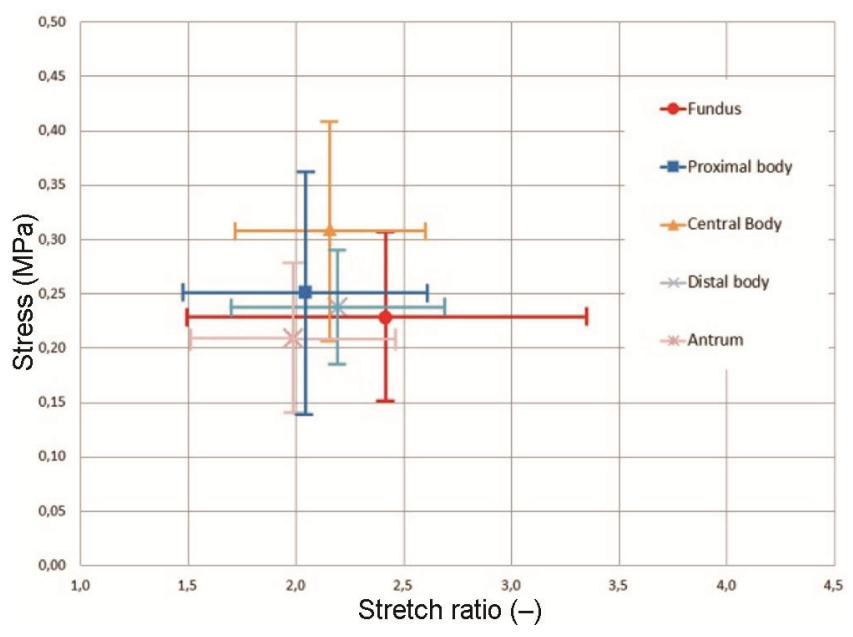

Figure 6. Average values of stress and stretch for main location on the stomach (fundus: $0.23 \pm 0.06 \mathrm{MPa}$ and $2.41 \pm 0.83$; proximal body: $0.25 \pm 0.11 \mathrm{MPa}$ and $2.05 \pm 0.55$; central body: $0.31 \pm 0.10 \mathrm{MPa}$ and $2.16 \pm 0.44$; distal body: $0.24 \pm 0.11 \mathrm{MPa}$ and $2.19 \pm 0.5$; antrum: $0.21 \pm 0.09 \mathrm{MPa}$ and $1.99 \pm 0.46)$. 


\section{RESEARCH COMMUNICATIONS}
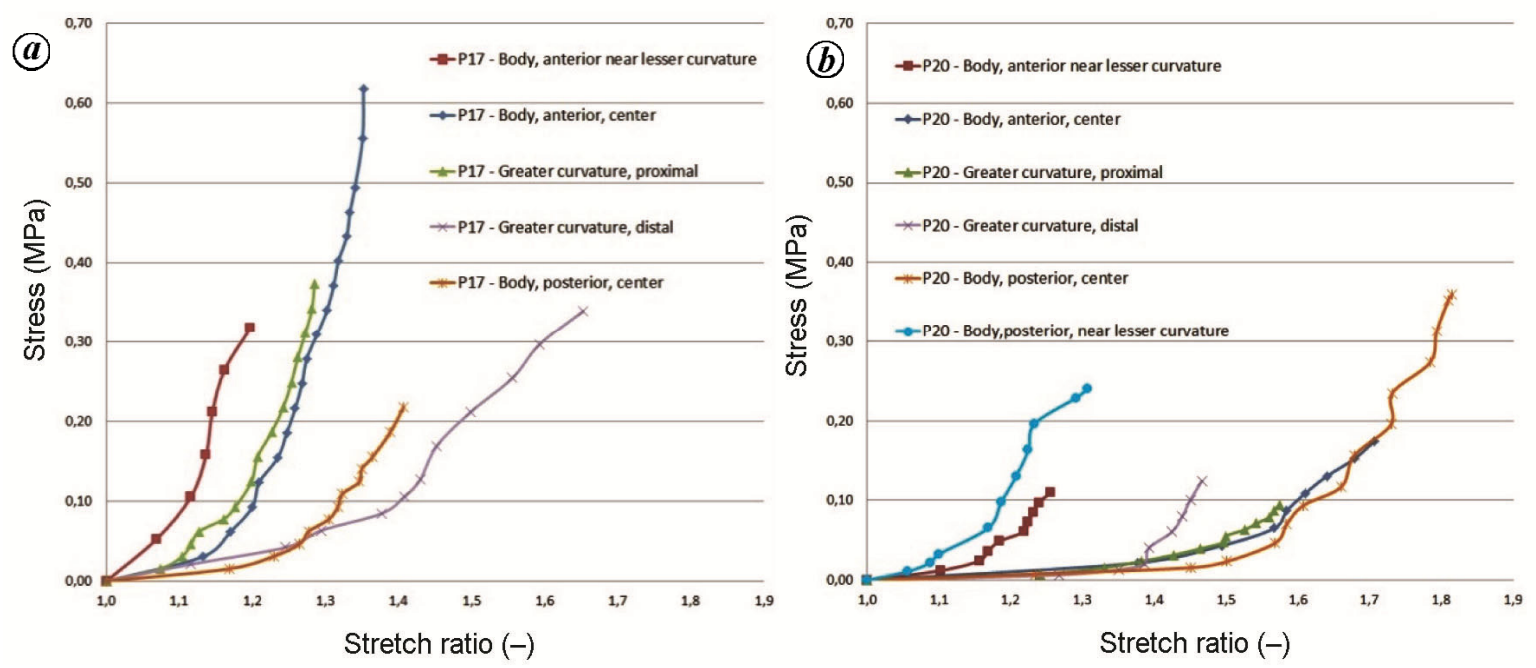

Figure 7. Stress-stretch ratio curves of test samples at different locations on two selected stomach specimens in the case of longitudinally cut samples: (a) from stomach no. 17, (b) from stomach no. 20.

tissue specimens demonstrated that stresses in the longitudinal muscle layer are $15-20 \%$ larger than in the circumferential test samples, but it could reach up to $50 \%$ for the submucosa-mucosa layer. It was also observed that the gastric mucosa and muscle layer determine the properties of the stomach wall ${ }^{17}$. Tensometric studies carried out on human tissues obtained from cadavers also demonstrated qualitatively varying mechanical properties in axial and transversal directions ${ }^{13}$, in which the values of maximum stress $(0.5 \mathrm{MPa})$ and maximum strain $(190 \%)$ were defined. The present study describes the mechanical properties of fresh human stomach specimens and provides novel, comprehensive data on the mechanical properties of the stomach wall with a detailed analysis of the anatomical regions of the stomach. The results from uniaxial stretching of samples excised from fresh human stomach specimens obtained during sleeve gastrectomy are presented. The tests were carried out to determine the fundamental mechanical properties of the examined tissues, in particular the maximum stress and elongation values and Young's modulus was estimated on this basis. Contrary to other studies ${ }^{8,9,13}$, test samples of varying dimensions (dependent on stomach size) were excised from the stomach specimens obtained in surgical procedures.

The conditions of the conducted tests differed from others previously described in the literature ${ }^{8,9,13,18}$. The tests were performed in atmospheric air (the stomach specimens were not submerged in saline solution bath nor sprayed during the tests). Organoleptic and visual quality assessment of the moisture level of test samples demonstrated that after the tests, the test samples did not display any signs of excessive dryness. The mucous membrane preserved an especially high moisture level. The material awaiting testing was stored in saline moistened gauze. The incised stomachs were not washed prior to testing.
The tests covered all of the stomach wall layers simultaneously. It was assumed that the studied stomach specimens exhibited properties corresponding to those of a living organ.

The examined stomach specimens were obtained from morbidly obese patients. Considering the dysregulated eating pattern of these patients, structural changes of the stomach wall are possible. Therefore, the results might not be representative of the general population. On the other hand, the main indication for stomach surgery nowadays is morbid obesity. Data on this particular clinical setting are required, even more than those for the general population.

The present study confirmed anisotropy of the mechanical properties of tissues of the stomach wall. Test samples cut transversely showed less stiffness and strength than those cut longitudinally. Heterogeneity of the tested material was demonstrated, because the properties of the test samples taken from different parts of the stomach specimen showed different levels of stiffness and strength. The maximum engineering stress of circumferential samples typically did not exceed $0.4 \mathrm{MPa}$, while for longitudinal samples the upper limit was $0.7 \mathrm{MPa}$. In both cases, results exceeding these values were observed, but they were probably caused by individual characteristics of the patients.

Mechanical differences across the sample can be related to the structure of the layered stomach walls and to the various physiological functions of each part of the stomach. Fibres of circular and longitudinal muscle layers are distributed in an irregular manner throughout the stomach, e.g. longitudinal layer clearly stands on small and the greater curvature of the stomach ${ }^{19,20}$. Moreover, test specimens were obtained from morbidly obese patients. Thus, the specimens were possibly affected by increased food consumption ${ }^{21}$ and obesity-related 
RESEARCH COMMUNICATIONS

Table 1. Stress-stretch ratio results (numbers in brackets correspond to those of the test samples

\begin{tabular}{llcc}
\hline Direction & \multicolumn{1}{c}{ Part of stomach } & $\sigma \pm$ SD & $\lambda \pm$ SD \\
\hline Transverse & Fundus (1) & $0.23 \pm 0.06$ & $2.41 \pm 0.83$ \\
& Proximal body (2) & $0.25 \pm 0.11$ & $2.05 \pm 0.55$ \\
& Middle body (3) & $0.31 \pm 0.10$ & $2.16 \pm 0.44$ \\
& Distal body (4) & $0.24 \pm 0.11$ & $2.19 \pm 0.50$ \\
& Antrum (5) & $0.21 \pm 0.09$ & $1.99 \pm 0.46$ \\
& & & \\
& Anterior, near lesser curvature (1) & $0.35 \pm 0.20$ & $1.72 \pm 0.50$ \\
& Anterior, centre (2) & $0.41 \pm 0.15$ & $1.67 \pm 0.47$ \\
& Greater curvature - proximal (3) & $0.32 \pm 0.17$ & $1.57 \pm 0.37$ \\
& Greater curvature - distal (4) & $0.39 \pm 0.26$ & $1.89 \pm 0.63$ \\
& Posterior, centre (5) & $0.34 \pm 0.13$ & $1.62 \pm 0.37$ \\
& Posterior, near lesser curvature (6) & $0.23 \pm 0.01$ & $2.00 \pm 0.70$ \\
\hline
\end{tabular}
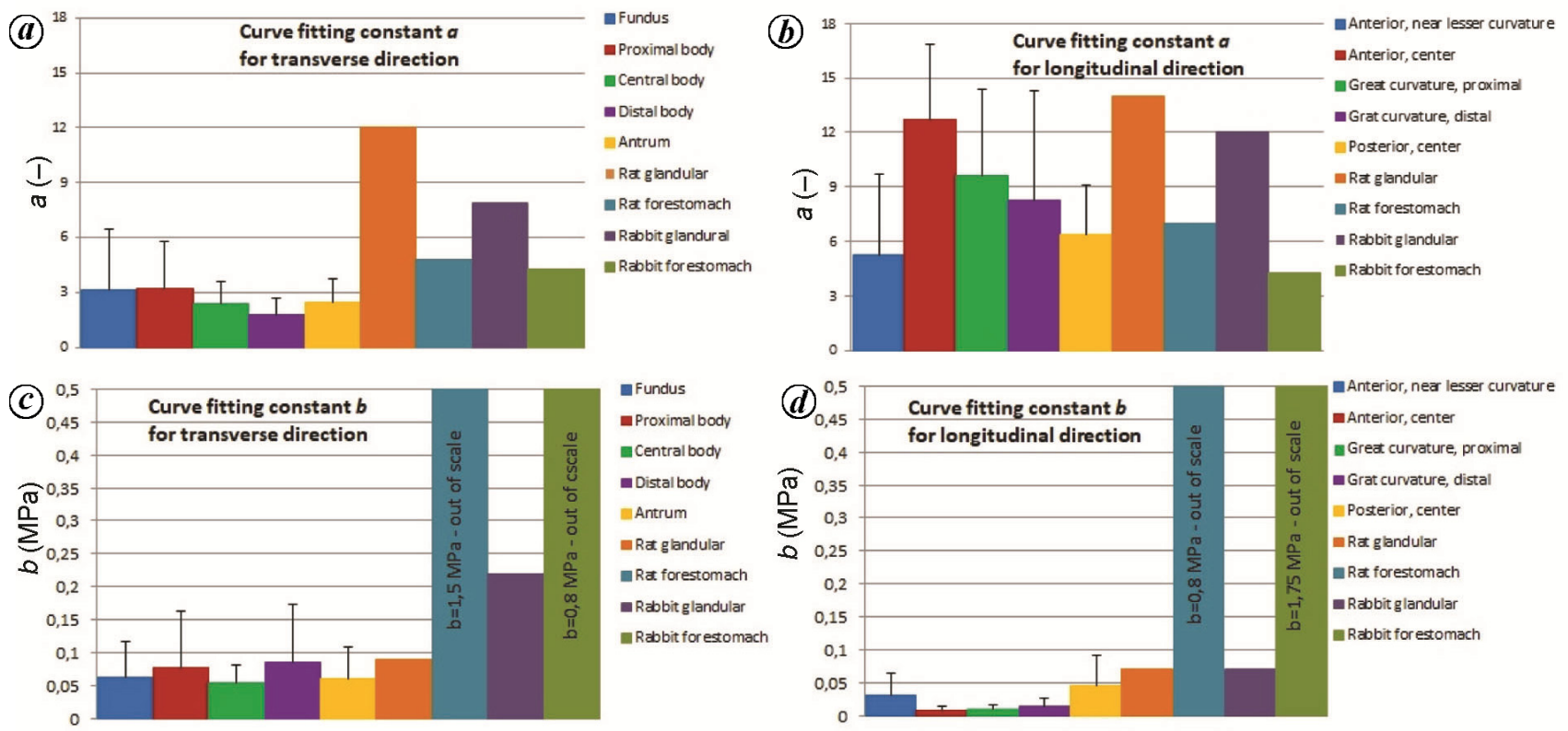

Figure 8. Comparison of $a$ and $b$ coefficients calculated in curve-fitting: $\boldsymbol{a}, a$ coefficient for transversely cut specimens; $\boldsymbol{b}, a$ coefficient for longitudinally cut specimens; $\boldsymbol{c}, b$ coefficient for transversely cut specimens; $\boldsymbol{d}, b$ coefficient for longitudinally cut specimens.

co-morbidities ${ }^{22-24}$. In the existing $\sigma-\lambda$ arrangement, the conducted tensile tests demonstrated a nonlinear and observable spread of mechanical properties among the test samples obtained from different parts of the stomach specimens. The observed nonlinearity can be approximated by exponential relation. Coefficients $a$ and $b$, which are calculated via curve fitting, adhere closely to the extension curves and can be easily used to define material properties in FEM applications as exponential variable.

The observed results of the tested parameters show a large spread of values between samples taken from the stomach of different donors, but cut from the same site (Figure 5) as well as samples from within one body (Figure 4). However, differences between the average values of the tested parameters of samples cut from the same location but from different organs do not present such a great spread (Figure 6). This information is crucial to represent the reality that physicians, researchers and design engineers need to overcome. In particular, engineers designing a new tool for a specific surgical problem based on overcoming mechanical resistance of tissues must ensure that the intended operation is performed with a single tool selected from the operating set. In addition to being of appropriate size, this tool must be able to withstand or overcome certain forces that may be contained within a relatively large range. For example, a force required to execute desired operations with the use of a specific device could be much higher or much lower than calculated from average values. The designer must ensure the possibility of using this device in the required ranges of force and to prevent it from exceeding these extreme values. An example of such types of tools is the stapler used during Laparoscopic Sleeve Gastrectomy (LSG). 
This equipment divides the operative stomach into two parts and seals the edges so that there is no leakage of contents of the stomach to the abdomen of a patient. Tightness guarantees staples piercing through the two layers of bonded tissues and curving on the other side, causing the material to compress. Clamping must be strong enough to achieve tightness, but at the same time it may not be too large due to the possibility of tissue necrosis in the seam and the ability to cut the tissue. In this sense, surgical tasks do not deviate from typical engineering tasks in which information about maximum or minimum values of mechanical properties is more important than their mean values. The apparent rate of leakage and bleeding after gastrectomy ${ }^{25,26}$ leads to the development of good practices ${ }^{27}$ in this surgical procedure where research into optimal tissue stapling could significantly improve operational standards. Nowadays, medical research is often supported by FEM calculations that allow for multiple simulations without the use of tissue material; yet reliable material data are required. Data from uniaxial tension, planar tension, biaxial tension and in some cases even triaxial tension studies ${ }^{9,28}$ are required for a full description of the material for FEM calculations. According to the authors, this work provides parts of such data, in the form of uniaxial tension characteristics. In spite of being limited to one dataset, which in fact is the simplest mechanical test, the results presented here carry a large dose of usability.

In conclusion, there are clearly noticeable differences in the strength and stiffness of the stomach wall, which are dependent on the direction of tension. The test samples which were stretched longitudinally (along the stomach length) were generally stiffer than the ones stretched transversely (across the stomach), because the strength of the longitudinal test samples was approximately $20 \%$ greater than in the case of the transverse test samples, and the stretch ratio was $20 \%$ lower in the longitudinal samples in comparison to the transverse samples. The measured properties are invaluable as input data for computer modelling of tissue behaviour, virtual reality surgical simulations and modelling of surgical techniques. The field of interest that can potentially benefit most from the obtained results is the modelling of alternative staple line configurations and examining them under loads specific to stomach after sleeve gastrectomy. Since tissues used in the present study have material properties close to those in a living stomach, the datasets can be used to simulate the behaviour of a 'living stomach' in a patient by means of FEA calculations in such applications as novel surgery procedures or in the design of medical equipment.

1. Brouwer, I., Ustin, J., Bentley, L., Sherman, A., Dhruv, N. and Tendick, F., Measuring in vivo animal soft tissue properties for haptic modeling in surgical simulation. Stud. Health Technol. Inf., $2001,81,69-74$.
2. Delingette, H. and Ayache, N., Soft tissue modeling for surgery simulation. In Computational Models for the Human Body (ed. Ciarlet, Ph.), Handbook of Numerical Analysis, Elsevier, 2004, pp. 453-550.

3. Rosen, J., Brown, J. D., De, S., Sinanan, M. and Hannaford, B., Biomechanical properties of abdominal organs in vivo and postmortem under compression loads. J. Biomech. Eng., 2008, 130(2), 021020; doi:10.1115/1.2898712.

4. Aurora, A. R., Khaitan, L. and Saber, A. A., Sleeve gastrectomy and the risk of leak: a systematic analysis of 4888 patients. Surg. Endosc., 2012, 26, 1509-1515; doi:10.1007/s00464-011-2085-3.

5. Sakran, N. et al., Gastric leaks after sleeve gastrectomy: a multicenter experience with 2834 patients. Surg. Endosc., 2013, 27, 240-245; doi:10.1007/s00464-012-2426-x.

6. Kobiela, J., Łaski, D. and Kaska, Ł., Detailed anatomy of staple line crossings in sleeve gastrectomies for morbid obesity. Surg. Obes. Relat. Dis., 2015, 11(6), 1404-1405.

7. Stroh, C. et al., Results of more than 11,800 sleeve gastrectomies: data analysis of the German Bariatric Surgery Registry. Ann. Surg., 2016; 263(5), 949-955; doi:10.1097/SLA.0000000000001559.

8. Zhao, J. B., Liao, D. H. and Gregersen, H., Tension and stress in the rat and rabbit stomach are location-and direction-dependent. Neurogastroenterol. Motil., 2005, 17, 388-398.

9. Fung, Y. C., Biomechanics. Mechanical Properties of Living Tissues, Springer, New York, USA, 1981.

10. Zhao, J., Liao, D., Chen, P., Kunwald, P. and Gregersen, H., Stomach stress and strain depend on location, direction and the layered structure. J. Biomech., 2008, 41, 3441-3447.

11. Pioletti, D. P., Rakotomanana, L. R. and Leyvraz, P.-F., Strain rate effect on the mechanical behaviour of the anterior cruciate ligament-bone complex. Med. Eng. Phys., 1999, 21, 95-100.

12. Manoogian, S. J., Bisplinghoff, J. A., McNally, C., Kemper, A. R., Santago, A. C. and Duma, S. M., Effect of strain rate on the tensile material properties of human placenta. J. Biomech. Eng., 2009, 131, 091008-(1-6).

13. Egorov, V. I., Schastlivtsev, I. V., Prut, E. V., Baranov, A. O. and Turusov, R. A., Mechanical properties of the human gastrointestinal tract. J. Biomech., 2002, 35, 1417-1425.

14. Ehrlein, H. J. and Schemann, M., Gastrointestinal Motility, Technical University of Munich, Germany.

15. Parkman, H. P., Training in gastrointestinal motility. Dig. Dis., 2006, 24, 221-227.

16. Schulze, K., Imaging and modelling of digestion in the stomach and the duodenum. Neurogastroenterol. Motil., 2006, 18, 172183.

17. Jia, Z. G., Li, W. and Zhou, Z. R., Mechanical characterization of stomach tissue under uniaxial tensile action. J. Biomech., 2015, 48, 651-658.

18. Yamada, H., Strength of Biological Materials, The Williams and Wilkins Company, Baltimore, USA, 1970.

19. Birmingham, A., The arrangement of the muscular fibres of the stomach. Trans. RAM Ireland, 1898, 16, 432; doi:10.1007/ BF03177287.

20. Dworken, H. J., Sircus, W., Keeton, W. T. and Hightower, N. C., Human digestive system, 2015; https://www.britannica.com/science/ human-digestive-system/Esophagus (accessed on 2 November 2016).

21. Geliebter, A., Gastric distension and gastric capacity in relation to food intake in humans. Physiol. Behav., 1988, 44, 665-668; doi.org/10.1016/0031-9384(88)90333-2.

22. Feldman, M. and Schiller, L., Disorders of gastrointestinal motility associated with diabetes mellitus. Ann Intern Med., 1983, 98, 378-384; doi:10.7326/0003-4819-98-3-378.

23. Yang, J., Zhao, J., Liao, D. and Gregersen, H., Biomechanical properties of the layered oesophagus and its remodelling in experimental type-1 diabetes. J. Biomech., 2006, 39, 894-904. 
24. Krishnan, B., Babu, S., Walker, J., Walker, A. B. and Pappachan, J. M., Gastrointestinal complications of diabetes mellitus. World $J$. Diab., 2013, 4(3), 51-63.

25. Gagner, M. and Buchwald, J. N., Comparison of laparoscopic sleeve gastrectomy leak rates in fourstaple-line reinforcement options: a systematic review. Surg. Obesity Relat. Dis., 2014, 10, 713-724; http://dx.doi.org/10.2147/MDER.S67338.

26. Ghosh, S. K., Roy, S., Chekan, E. and Fegelman, E. J., A narrative of intraoperative staple line leaks and bleeds during bariatric surgery. Obes. Surg., 2016, 26(7), 1601-1606; doi:10.1007/s11695016-2177-1.

27. Rosenthal, R. J., International sleeve gastrectomy expert panel consensus statement: best practice guidelines based on experience of $>12,000$ cases. Surg. Obes. Relat. Dis., 2012, 8, 8-19.

28. Sacks, M. S. and Sun, W., Multiaxial mechanical behavior of biological materials. Annu. Rev. Biomed. Eng., 2003, 5, 251-284; doi:10.1146/annurev.bioeng.5.011303.120714.

ACKNOWLEDGEMENT. This study was supported by the MN 010111/08 grant provided by the Medical University of Gdansk, Poland.

\section{Engendering finger millet-based value chains for livelihood and nutritional security of women in agriculture}

\author{
J. Charles Jeeva ${ }^{1, *}$, Kushagra Joshi ${ }^{2}$, \\ Abha Singh ${ }^{1}$ and B. C. Behera ${ }^{1}$ \\ ${ }^{1}$ ICAR-Central Institute for Women in Agriculture, \\ Bhubaneswar 751 003, India \\ ${ }^{2}$ ICAR-Vivekananda Parvatiya Krishi Anusandhan Sansthan, \\ Almora 263 601, India
}

Gender analysis along the agricultural value chain enhances our understanding about the roles performed by men and women in the value chain and in forward and backward linkages, their access to productive resources, opportunities available for valueaddition and benefit sharing, both as individuals and group enterprises. The gender roles in finger milletbased value chains and the constraints faced by women in existing finger millet value chains in Tamil Nadu, Karnataka and Odisha have been documented in this study. Along the value chains, women were found only as farmers, petty traders or labourers in processing units. However, the entry points for women in finger millet value chain have been identified. The role of women in the value chain could be strengthened by mobilizing them into clusters, establishing milletprocessing units, and forming forward and backward linkages.

\footnotetext{
*For correspondence. (e-mail: jcjeeva@gmail.com)
}

Keywords: Gender, finger millet, value chain, livelihood, nutritional security.

GENDER analysis along agricultural value chain enhances our understanding about the roles performed by men and women in the value chain. Secondary agriculture, more specifically the value chain development in agriculture provides a great opportunity for income enhancement, and it is very significant to recognize the position of women in the value chain for their socio-economic empowerment. Enhancing the participation of women in agricultural value chains will not only enhance their purchasing powers, but will also empower them by allowing participation in decision-making and access to resources.

Explicitly gendered studies of value chains have mostly been carried out with reference to the horticultural sector $^{1,2}$. Against this backdrop, the present study has been conducted with the objectives to examine and document the gender roles in finger millet (ragi)-based value chains, to study gender-based constraints faced by women and to identify entry points to strengthen their role in these value chains by forming forward and backward linkages.

The finger millet-based value chain has been selected for the present study, as the crop is considered as propoor, pro-nutrition and pro-women in nature. Secondary data on state-wise production of finger millet in Indian markets, different value-added products and the technologies available for its production and processing were collected. On the basis of this information, states were selected, markets identified, research instruments developed, technologies identified for action research and value-added products to be upscaled were also identified. The research instruments required for the study, i.e. the questionnaires for value-chain mapping and interview schedules for each value-chain actor were prepared on the basis of extensively collected secondary literature. Focused group discussions and in-depth household surveys were also conducted to collect the required data. The technology used in millet production, post-harvest processing, value-addition, nodes in the marketing chain and the role of women in different nodes along the value chain were studied. The sample size was 120 stakeholders contacted through snow-ball sampling technique, representing the farmer producers, retailers, wholesale traders and processors from Koraput district of Odisha, Tumkur district of Karnataka and Madurai district of Tamil Nadu (TN).

The channels for finger millet were mapped by visiting markets in the study area. Major functions mapped were input supply, production, processing, trading and consumption. Instead of starting with the farmers/production node, backward mapping of the chain was followed, i.e. the mapping process started with the industry involved in processing and marketing of value-added products of finger millet. Figure 1 shows the gendered roles in finger 